\title{
Contrast Enema for Hirschsprung Disease Investigation: Diagnostic Accuracy and Validity for Subsequent Diagnostic and Surgical Planning
}

\author{
Giovanni Frongia ${ }^{1}$ Patrick Günther ${ }^{1} \quad$ Jens-Peter Schenk ${ }^{2} \quad$ Kai Strube $^{1}$ Markus Kessler $^{1}$ \\ Arianeb Mehrabi ${ }^{3}$ Philipp Romero ${ }^{1}$
}

${ }^{1}$ Division of Pediatric Surgery, Department of General, Visceral and Transplantation Surgery, University Hospital of Heidelberg, Heidelberg, Germany

2 Division of Pediatric Radiology, Department of Diagnostic and Interventional Radiology, University Hospital of Heidelberg, Heidelberg, Germany

3 Department of General, Visceral and Transplantation Surgery, University Hospital of Heidelberg, Heidelberg, Germany

Eur J Pediatr Surg 2016;26:207-214.

\author{
Address for correspondence Giovanni Frongia, MD, Division of \\ Pediatric Surgery, Department of General, Visceral and \\ Transplantation Surgery, University Hospital of Heidelberg, Im \\ Neuenheimer Feld 110, Heidelberg 69120, Germany \\ (e-mail: gio@frongia.net).
}

\begin{abstract}
Keywords

- Hirschsprung disease

- contrast enema

- rectal biopsy

- diagnostic accuracy

Introduction A targeted Hirschsprung disease (HD) diagnostic is necessary, as it determines a specific approach primarily based on surgical resection of the affected aganglionic colonic segment. The aim of this study was to evaluate the diagnostic accuracy of a contrast enema (CE) for HD diagnosis and to determine whether it should be performed before or after rectal biopsies (RBs).

Methods A retrospective observational study of children undergoing RB for HD investigation was performed. In the performed $\mathrm{CE}$, the occurrence and the level of a colonic caliber change (CCC) were recorded and its concordance with the histologically assessed level of aganglionosis by RB and the odds ratio were calculated.

Results A total of 107 cases were included. Sensitivity and specificity for a CCC in CE were $74.1 \%$ and $94.6 \%$. A CCC present in CE was associated with a 50 -fold increased probability for a histologically proven $\mathrm{HD}$. The overall concordance between a CCC and the histologically assessed level of aganglionosis was high (kappa 0.642, $p=0.003$ ), being correct in $94.4 \%$ of cases when the CCC was located in the rectosigmoid, but only in $50 \%$ of cases when it was located in more proximal segments. By performing a CE only after HD diagnosis confirmation by RB would avoid $67.5 \%$ of CE with no loss of diagnostic accuracy.

Conclusion We confirm that CE is a valuable tool for HD diagnosis; however, it should only be performed for subsequent diagnostic and surgical planning following histological confirmation of HD by RB. On the basis of this, an algorithm for an optimized investigation and management of $\mathrm{HD}$ is presented.
\end{abstract}

(c) 2016 Georg Thieme Verlag KG Stuttgart · New York
DOI http://dx.doi.org/ 10.1055/s-0035-1546755. ISSN 0939-7248. 


\section{Introduction}

Hirschsprung disease (HD) is characterized by colonic aganglionosis leading to chronic constipation and can be specifically treated by resection of the affected aganglionic segment and the preceding hypoganglionotic segment. ${ }^{1,2}$ Therefore, a targeted HD diagnostic is necessary. Three different approaches for diagnosing HD are available: contrast enema (CE), anorectal manometry, and rectal biopsy. In CE the presence of a change in colonic caliber and in anorectal manometry the absence of a rectosphincteric reflex are possible, but not definite signs of HD. The decisive and gold standard method for HD diagnosis is the histological confirmation of absent ganglionic cells as well as the detection of acetylcholinesterase-positive hypertrophic nerve fibers in colonic biopsies. ${ }^{1,3-5}$ The surgical management of HD depends on an unambiguous histological diagnosis of aganglionosis and an accurate assessment of the degree of aganglionosis. ${ }^{6-9}$ The level of colonic caliber change in a CE can help to predict the degree of aganglionosis and therefore the length of the colonic segment to be resected and whether laparoscopic assistance during surgery is necessary or an open colectomy is indicated, such as for total colonic aganglionosis. Our study aimed to evaluate the diagnostic accuracy of CE for HD investigation and its validity for subsequent diagnostic steps and surgical planning in our cohort of chronic constipated children. Therefore, we first evaluated the impact of $\mathrm{CE}$ performed before rectal biopsy in our current diagnostic procedure and, in a second step, the impact of performing a CE only in cases of HD diagnosis after histological proof by rectal biopsy, as suggested by Martucciello et al. ${ }^{4}$

\section{Patents and Methods}

\section{Study Design}

Approval for the study was obtained from the institutional research ethics board. A retrospective cohort study was performed by chart review over an 11-year period (January 1 , 2003, to December 31, 2013) of all children undergoing nearfull-thickness rectal biopsy for the investigation of HD at our pediatric surgery tertiary referral center. In this study, we excluded patients revaluated for constipation after a resection procedure for $\mathrm{HD}$, patients with other previous intestinal operation, or patients with additional diagnoses causative for constipation.

\section{Diagnostics}

HD diagnosis was determined by the absence of ganglion cells and the presence of acetylcholinesterase-positive hypertrophic nerve fibers in rectal biopsies, ${ }^{1,3,5}$ as reported by the pathology reports obtained from a national reference pathology center for HD. One biopsy was obtained at $5 \mathrm{~mm}$ and further at different levels for 1 to $4 \mathrm{~cm}$ above the dentate line. CE was performed by pediatric radiologists in a standardized procedure. Any laxative preparation was stopped at least 1 to 2 weeks before the investigation. Until the age of 6 months a non-ionic, iodine contrast medium was used (Imeron 300, Bracco Imaging, Konstanz, Germany), while after the 6th month of age a barium-containing contrast medium was used (Micropaque, Guebert, Sulzberg, Germany). The contrast agent was instilled under radiological control in anteroposterior and lateral view at least until the left colonic flexure was reached. A delayed X-ray image was obtained six or more hours after the contrast enema. All CE were reviewed by the head of pediatric radiology department (SJP) blinded to the clinical outcome of the patients. The presence and level of colonic caliber change in the CE was recorded. - Fig. 1 presents a CE with a representative caliber change at the level of the rectosigmoid reconcilable with a HD. The rectosigmoid index was calculated as the widest diameter of the rectum divided by the widest diameter of the sigmoid colon. A rectosigmoid index $<1$ is defined as pathological indicating a HD. ${ }^{10,11}$

\section{Concordance Study}

We assessed the radiohistological concordance of the level of aganglionosis in those patients where a CE and resection of aganglionic colon were performed. The levels of aganglionosis were subdivided into two groups according to CE and histology: "within rectosigmoid segment" and "proximal to rectosigmoid segment," as this differentiation has clinical relevance for the selection of the surgical approach, such as colonic resection with or without laparoscopic assistance. Then the Kappa coefficient was calculated between the levels of caliber change in the CE and the histologically detected level of aganglionosis in the surgical resection specimen. ${ }^{12}$

\section{Impact of Modified Diagnostic Pathway}

In our clinic, the current diagnostic approach in chronic constipated children suspected for HD involves at first a CE followed by near-full-thickness rectal biopsies in most cases. We virtually modified the order of our diagnostic procedures according to the order suggested in the literature ${ }^{4}$ involving at first rectal biopsy for a conclusive investigation for HD, subsequently followed by a CE only in cases of previously histologically proven $\mathrm{HD}$, and assessed the impact of these changes.

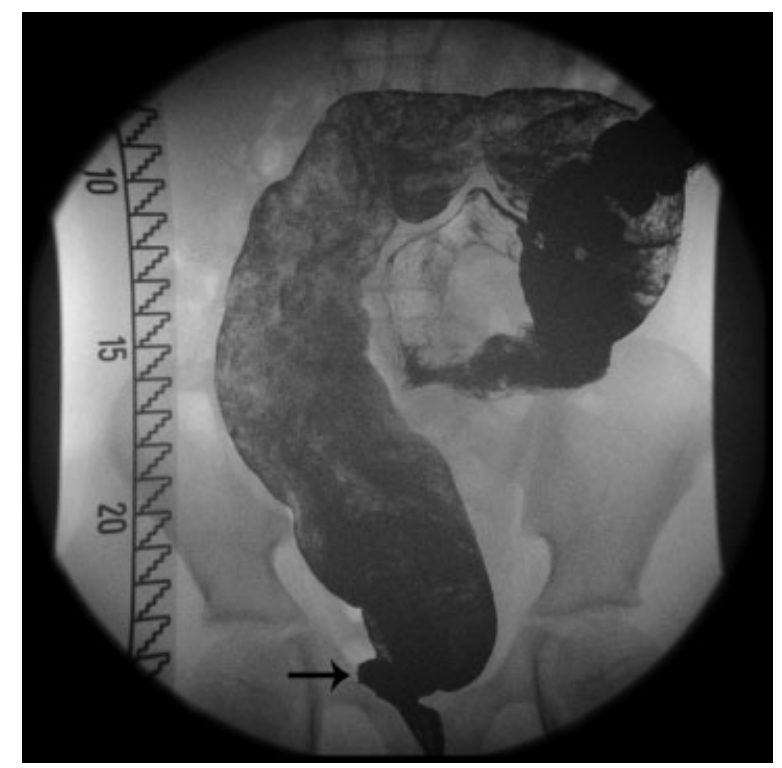

Fig. 1 Contrast enema with a representative caliber change at the level of the rectosigmoid (arrow) reconcilable with an HD. 
Table 1 Patients' characteristics

\begin{tabular}{|l|l|l|l|}
\hline & HD & Non-HD & $p$ \\
\hline Patients $(n)$ & 32 & 75 & \\
\hline Age (months, mean) & 15.7 & 59.6 & $<0.00001$ \\
\hline$<1$ mo $(n)$ & 5 & 0 & 0.02 \\
\hline$>12$ mo $(n)$ & 8 & 61 & $<0.000001$ \\
\hline Sex $(n)$ & \multicolumn{5}{|l|}{} & 0.018 \\
\hline Male:female ratio & $25: 7$ & $40: 35$ & \\
\hline
\end{tabular}

\section{Data Analysis}

The statistical analysis was performed using SPSS Version 18 for Windows (SPSS Corp., Chicago, Illinois, USA). An independent-samples $t$-test or a Fisher exact test was conducted as appropriate to compare the diagnosis of HD with other parameters. The concordance study was performed by calculating the Kappa coefficient, which is a statistical measure of interrater agreement for categorical items. As it takes into account the agreement occurring by chance, it is thought to be a more robust measure than simple percent agreement calculation. Acceptable values of Kappa for the comparison of two groups were as follows: 0 to 0.20 : very low agreement; 0.21 to 0.40 : low; 0.41 to 0.60 : moderate; 0.61 to 0.80 : full; and 0.81 to 1 : almost perfect. ${ }^{12}$ Univariate logistic regression analysis was performed to determine the odds of a radiographic predictor for a histologically proven HD. A $p<0.05$ was considered significant.

\section{Results}

\section{Patient Characteristics}

During the study period, 128 children with a history of chronic constipation underwent near-full-thickness rectal biopsy for investigation of HD. We excluded 21 (16.4\%) patients from this study due to previous bowel surgery ( $n=16$ ), other diagnosis (Currarino syndrome $n=1$, desmosis coli $n=1$, anal atresia $n=1$; chronic intestinal pseudoobstruction $n=1$ ) or unavailability of charts and CE radiographs $(n=1)$. - Table 1 presents the demographic data of the 107 included patients. Patients were assigned to the HD or non-HD group, depending on the pathology result of the rectal biopsy. In the HD group, the mean age was significantly lower compared with the non-HD group. HD was diagnosed significantly more often within the first year of life. Males were diagnosed with HD significantly more often, with a female-to-male ratio of $\sim 1: 3.5$.

\section{Radiological and Histological Findings}

-Fig. 2 presents the radiological and histological findings in our cohort. Of the 107 included children, a CE was performed in 83 cases (77.6\%). In 23 cases (27.7\%), the CE presented a colonic caliber change (CCC). Of these, 20 cases (87\%) had a histologically assessed aganglionosis by rectal biopsy. In the remaining 60 cases without a CCC in the $\mathrm{CE}$, an HD was assessed histologically by rectal biopsies in 7 cases (11.7\%). In conclusion, in the present series, CE sensitivity was $74.1 \%$, specificity $94.6 \%$, false-positive rate $25.9 \%$, and false-negative rate $5.4 \%$.

-Table 2 presents the radiographic findings from CE in detail. A CE was performed with similar frequency in each group ( $p=0.321)$. A CCC in the CE was observed more frequently in the HD group than in the non-HD group $(p<0.000001)$. The mean rectosigmoid index was lower $(p=0.498)$ and more often pathological $(p=0.03)$ in the HD group. However, only $25.9 \%$ of the overall patients in the HD group had a pathological rectosigmoid index. In the present series the rectosigmoid index sensitivity was $77.8 \%$, specificity $75 \%$, false-positive rate $22.2 \%$, and false-negative rate $25 \%$.

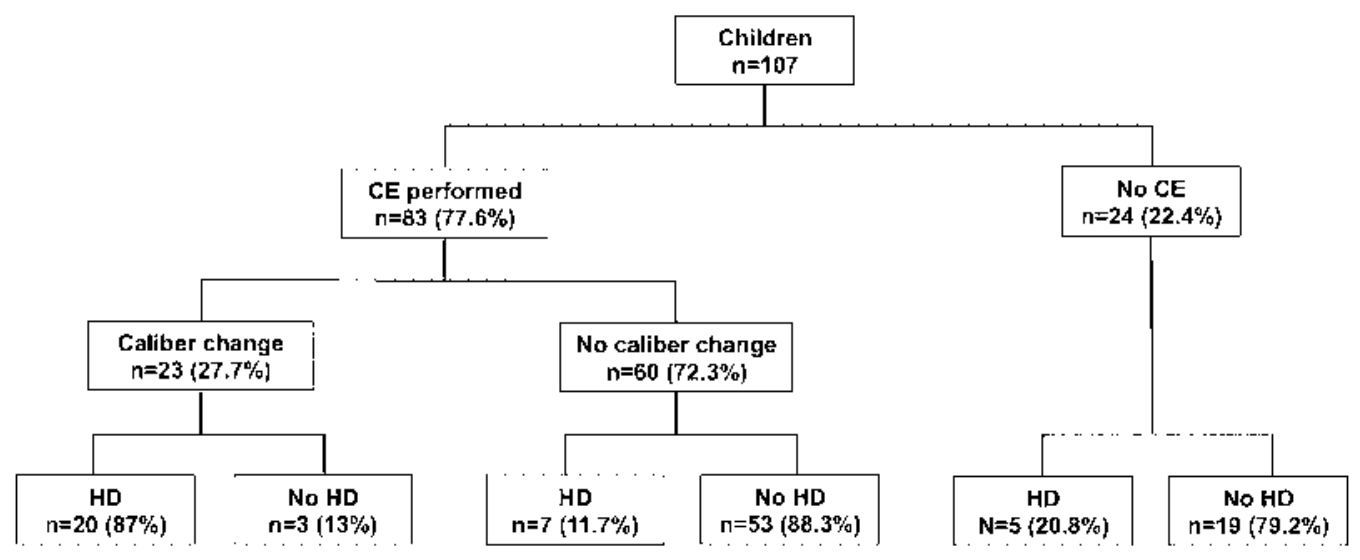

Fig. 2 Radiological findings in contrast enema (CE) and histological findings in subsequent biopsies. 
Table 2 Radiological findings

\begin{tabular}{|l|l|l|l|}
\hline \multicolumn{1}{|l|}{} & HD & Non-HD & $p$ \\
\hline Patients $n$ & 32 & 75 & \\
\hline Contrast enema $n(\%)$ & $27(84.4 \%)$ & $56(74.7 \%)$ & 0.321 \\
\hline Performed & $20(74.1 \%)$ & $3(5.3 \%)$ & $<0.000001$ \\
\hline Colonic caliber change present & & \multicolumn{3}{|l|}{} \\
\hline Rectosigmoid index & 0.96 & 2.51 & 0.498 \\
\hline Mean & $7(25.9 \%)$ & $2(3.6 \%)$ & 0.03 \\
\hline Pathological $n(\%)$ & &
\end{tabular}

Radiological Predictors of Histologically Confirmed HD -Table 3 presents the binary univariate logistic regression results for radiological signs in $\mathrm{CE}$ as predictors of a histologically proven HD diagnosis by rectal biopsy. The presence of a change of colonic caliber in a CE was associated with a 50-fold increased probability of a histologically confirmed HD diagnosis (odds ratio [OR], 50.48; 95\% confidence interval [CI], $11.88-214.54 ; p<0.000001$ ), while a pathologic rectosigmoid index of $<1$ was not associated with a higher probability of histological HD diagnosis (OR, 0.1; 95\% CI, 0.018-0.51; $p=0.006)$. A CE performed before the age of 30 days had a sevenfold higher probability of false-negative results (OR, 7.2; 95\% CI, 1.05-49.32; $p=0.044)$.

\section{Concordance Study}

-Table 4 shows the comparison between the level of aganglionosis determined by change of colonic caliber detected in CE and the level of aganglionosis histologically proved by evaluation of the surgically resected specimen. In total, 19 patients who underwent both $\mathrm{CE}$ and colonic resection were studied. The overall level of concordance between both methods to assess the level of aganglionosis was high (kappa $0.642, p=0.003$ ) and matched in 18 of 19 patients (95\%). Performing the analysis of concordance after stratifying the level of aganglionosis in "within" and "outside" the rectosigmoidal segment shows that a change of colonic caliber present in a CE within the rectosigmoidal segment predicted accurately the histologically assessed level of aganglionosis in approximately $95 \%$ of cases. In contrast, when the change of colonic caliber was present outside of the rectosigmoid segment, it predicted correctly the histologically assessed level of aganglionosis in only $50 \%$ of cases.

\section{Impact of Modified Diagnostic Pathway}

In our current diagnostic pathway, HD investigation was started with a CE in 83 children. - Fig. 3 presents the outcome occurring if our diagnostic pathway would be virtually modified into initially performing rectal biopsies for HD investigation and CE would be only performed in children with prior histologically proven HD as suggested by Martucciello et al. ${ }^{4}$ In this modified approach, a CE would have been only performed in 27 children, thus only in $32.5 \%$ of cases, without missing any case of HD by using this modified approach.

Furthermore, in the current approach, 63 of the $83 \mathrm{CE}$ (75.9\%) did not add any valuable information, because after $\mathrm{CE}$, children had no histological proof of $\operatorname{HD}(n=56)$ or despite histological HD diagnosis the CE did not show a change of colonic caliber $(n=7)$. In contrast, in the modified approach, only 7 of the 27 performed CE (25.9\%) would not show a change of colonic caliber and, therefore, did not add any valuable information regarding the proximal level of aganglionosis.

In five cases in our cohort, rectal biopsies were all aganglionic up to the highest biopsy. In these cases, a long-segment aganglionosis was possible; therefore, after

Table 3 Binary univariate logistic regression of radiological predictors in CE of a histological proven HD

\begin{tabular}{|l|l|l|l|l|l|}
\hline & OR & 95\% Cl & $P$ \\
\hline Change in colonic caliber present & 50.48 & 11.88 & - & 214.54 & $<0.000001$ \\
\hline Pathological rectosigmoid index $<1$ & 0.10 & 0.018 & - & 0.51 & 0.006 \\
\hline
\end{tabular}

Abbreviations: $\mathrm{CE}$, contrast enema; $\mathrm{Cl}$, confidence interval; $\mathrm{HD}$, Hirschsprung disease; OR, odds ratio.

Table 4 Accuracy of a contrast enema (CE) in predicting the level of aganglionosis

\begin{tabular}{|c|c|c|c|}
\hline & CE & Histology & Incorrect prediction in CE \\
\hline \multicolumn{4}{|l|}{ Level of aganglionosis } \\
\hline Within rectosigmoid & 18 & 17 & $5.6 \%$ \\
\hline Proximal to rectosigmoid & 1 & 2 & $50 \%$ \\
\hline
\end{tabular}




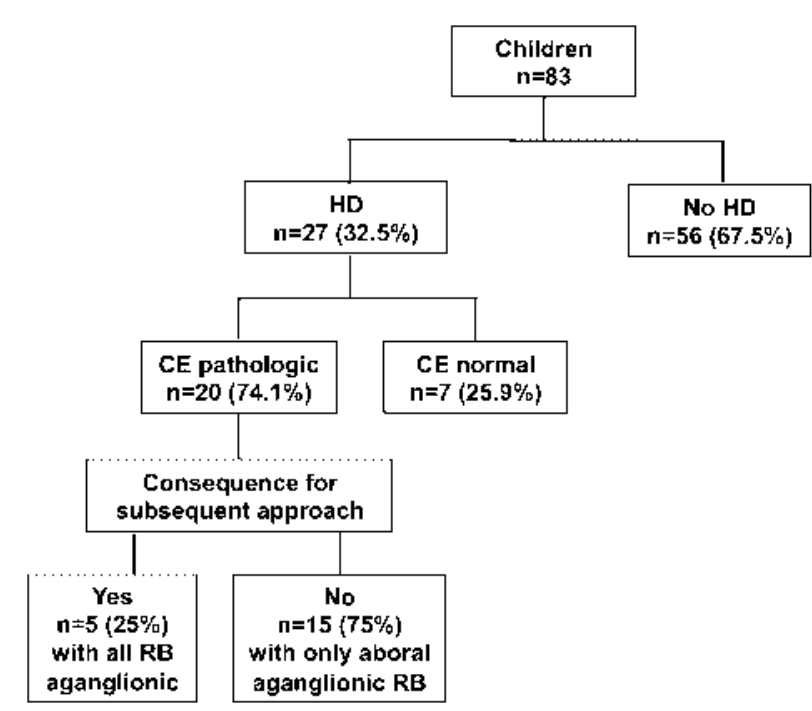

Fig. 3 Modified diagnostic pathway: findings and consequences for subsequent approach in $n=83$ children who had both rectal biopsy and contrast enema (CE).

histological HD diagnosis, the $\mathrm{CE}$ was analyzed for the presence of a change in colonic caliber. This analysis revealed a change in colonic caliber present within the rectum $(n=2)$, sigma $(n=1)$, and descending colon $(n=1)$, while in one case the CE was inconclusive. Laparoscopic $(n=4)$ or open $(n=1)$ performed colonic biopsies confirmed in all cases the level of aganglionosis suggested by the level of change in colonic caliber in the $\mathrm{CE}$. In the individual case of inconclusive $\mathrm{CE}$, the colonic biopsy showed a level of aganglionosis at the left colonic flexure. Transanal-Endorectal Pull-Through (TERPT) ${ }^{13}$ was performed in these five cases, of which three cases with laparoscopic assistance (level of aganglionosis in descending colon in two cases and in sigmoid colon in the other) (-Fig. 3).

\section{Discussion}

A reliable diagnosis of HD is crucial as it determines a specific approach, primarily based on surgical resection of the affected aganglionic colonic segment. Martucciello et al provided diagnostic guidelines for HD and underlined the importance of initial rectal biopsy to achieve the diagnosis, whereas $C E$ should be limited to the prediction of the proximal level of aganglionosis to plan surgical approach. ${ }^{4}$ Other authors approved that rectal biopsies alone can confirm the HD diagnosis sufficiently for surgical indication due to its considerably higher specificity and sensitivity compared with $C E^{1-3,14}$ and that $C E$ might subsequently be useful in selecting the operative approach. $3,7,15$

In our clinic, we initially perform a CE as it is largely considered an appropriate initial screening tool for HD in children $^{3,15-17}$ and the current national guidelines for HD investigation do not define any specific chronologic order of diagnostic procedure. ${ }^{18}$

However, by following the suggested modified approach in our cohort, we would have avoided $67.5 \%$ of all CE and reduced the percentage of $\mathrm{CE}$ without any valuable information regarding the proximal level of aganglionosis to $25.9 \%$. Therefore, we would not only reduce the number unnecessary CE for HD diagnosis but also reduce the number of misleading $\mathrm{CE}$, aiding a more stringent indication for $\mathrm{CE}$ without compromising accuracy in HD diagnostic.

While in the CE the evaluation of the rectosigmoid index is not helpful for HD investigation, the presence of a CCC in the CE was significantly associated with a 50-fold increased probability for a histologically proven HD. Therefore, if a CCC in the CE is present, it is a good predictor of HD. However, this radiological sign has some clear limitations. We found out that the potential of the caliber change to radiologically correctly predict the level of aganglionosis strongly depends upon the extension of the HD. Particularly, it is best suitable for this purpose when the aganglionosis is located within the rectosigmoid segment ( $~ 75 \%$ of HD patients), but might fail to be reliable in $\sim 15 \%$ of HD patients with long-segment HD, therefore, with aganglionosis of the descending, transverse, and/or ascending colon. Furthermore, when CE was performed in the first 30 days of life, we found a sevenfold higher probability for false-negative results and can confirm the low reliability of $\mathrm{CE}$ when performed in neonates reported by others. ${ }^{9,19-22} \mathrm{~A}$ possible reason for this might be that a colonic dilatation, noticeable as caliber change in the $\mathrm{CE}$, needs a certain time to develop before it can be detected in a CE and, thus, might not be visible in the first month of life.

In conclusion, a CE might be a helpful, but certainly not an optimal initial diagnostic tool for HD. In particular, the extent of aganglionosis and the age of the patient are limiting factors for a reliable diagnosis in a CE. The more reliable and decisive method for HD diagnosis is the more invasive histological confirmation of HD in colonic biopsies. ${ }^{1,3-5}$ However, the prevalence of chronic obstipation in the Western pediatric population ranges between 2 and $5 \%$, while HD is a rare disease with an incidence of 1:5000. Therefore, not all children with constipation symptoms should be subjected to rectal biopsies, as there would be significant consequences to the pediatric health care systems but only a few HD diagnoses. As a consequence, the indication criteria for rectal biopsies must be strict and reserved for a subgroup of chronic constipated children in which the probability for a HD is high. This subgroup might be selected on the basis of their medical history (i.e., delayed meconium passage more than $48 \mathrm{~h}$ after birth, episodes of enterocolitis, significant constipation refractory to treatment, onset of constipation in infancy, particularly after onset of food supplement and before toilet training, older children with failure to thrive or very untypical, intractable constipation) and physical examination (abdominal distension). ${ }^{3,23}$ On the basis of our data, for this subgroup of chronic constipated children, we underline the importance of initial rectal biopsy for HD diagnosis, whereas CE should be limited to the prediction of the proximal level of aganglionosis to plan surgical approach. In chronic constipated children not meeting these criteria a HD is seldom and a CE is further justified as an initial diagnostic to rule other causes of chronic constipation, while for them the indication for rectal biopsies for HD investigation must be established 
individually. However, in neonates, a CE is not only performed for HD diagnosis, but also for meconium ileus, small left colon syndrome, or diagnosis of bowel atresia and stenosis. Therefore, $\mathrm{CE}$ is a valuable tool in neonates when the clinical diagnosis is not specific.

Rectal specimen for histological HD evaluation can be achieved by suction biopsy or by near-full-thickness rectal biopsies. The complication rates for both methods are low and both are reported to be safe procedures. ${ }^{24-26}$ The rectal suction biopsy method is the current gold standard in the histological diagnosis of HD, with the advantage of a higher availability in the in the clinical setup as it does not require general anesthesia as needed for near-full-thickness rectal biopsies. ${ }^{4,23}$ However, suction biopsy only contains submucosal plexus, ${ }^{27,28}$ while near-full-thickness rectal biopsies include also the myenteric plexuses in the specimen available for histological evaluation. This can make the difference in safely ruling out a very short HD segment. For this purpose, one biopsy should be taken as close as possible to the dentate line ( $\sim 5 \mathrm{~mm}$ above), without being within this physiological hypoganglionosis zone. This zone reaches different levels above the dentate line: $10-25 \mathrm{~mm}$ for submucosal, while only $5 \mathrm{~mm}$ for the myenteric plexus. ${ }^{28-30}$ As the near-fullthickness rectal biopsies also contain the myenteric plexus, they can be taken closer to the dentate line, and can therefore diagnose a very short segment HD more reliably than suction biopsies. ${ }^{28-30}$ Furthermore, 9 to $17 \%$ suction biopsies are reported to be inadequate by most large series, principally due to insufficiently included submucosa, which increases the risk for false diagnosis and the necessity to rebiopsy. ${ }^{24,28,31,32}$ A further disadvantage of suction biopsies is the limited size of obtainable specimen and the difficulty of accurate positioning of the suction biopsy device, especially in a moving child. ${ }^{28}$ Both issues are better approached by a near-full-thickness biopsy under general anesthesia. Therefore, we perform near-full-thickness rectal biopsies; however, the rectal specimen can also be obtained by suction biopsy, as this is the current gold standard in the histological diagnosis of HD. ${ }^{4,23}$

If HD probability is high, based on the aforementioned medical history and physical examination criteria, for HD diagnosis and subsequent management we suggest the algorithm shown in -Fig. 4, which is based on the literature and present data. This algorithm puts rectal biopsy (near-fullthickness rectal or suction) in the first instance of the HD diagnostic, with different scenarios possible after rectal biopsies. If the rectal biopsies are only in part aganglionic and consequently confirm an aganglionosis limited to the rectosigmoid, a CE is dispensable and a Transanal-Endorectal Pull-Through procedure (TERPT) ${ }^{13}$ can be performed. An intraoperative histological evaluation of the extent of aganglionosis is always mandatory to confirm a complete resection of the aganglionic segment. ${ }^{4,14}$ If all biopsies are aganglionic, the extent of aganglionosis cannot be assessed by rectal biopsies. In these cases, a CE is the only noninvasive routine exploration at the surgeon's disposal to assess the level of aganglionosis and plan the corrective surgery. If the $\mathrm{CE}$ shows a caliber change within the sigmoid segment, one might proceed to a TERPT, as our data show a high concordance between the radiological and histological assessed level of aganglionosis within this segment, which was confirmed by others. $6,33,34$ In cases the caliber change is located proximal to the

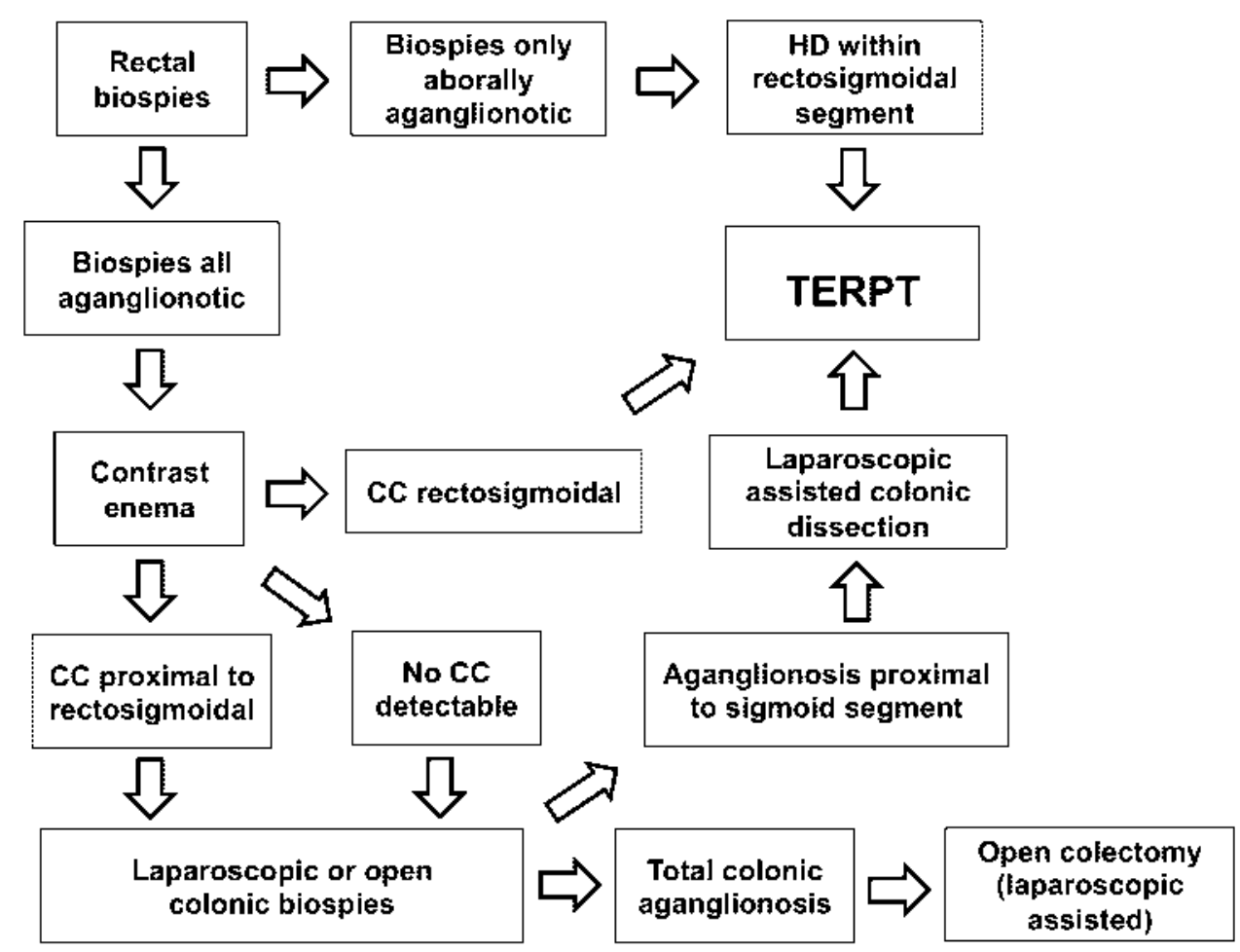

Fig. 4 Suggested algorithm to HD investigation and surgical management. CC, caliber change in contrast enema; TERPT, Transanal-Endorectal Pull-Through operation. 
rectosigmoid segment, we support further histological assessment of the proximal level of aganglionosis by laparoscopic biopsies, as we could show that the level of caliber change in CE is not reliable when located proximal to the rectosigmoid in accordance with others. ${ }^{6,33}$ In case the performed CE does not show any caliber change, we suggest also performing additional laparoscopic colonic biopsies to rule out a total aganglionosis in accordance with others. ${ }^{17,33-35}$ A confirmed total colonic aganglionosis gives the indication for ileostomy and (laparoscopic-assisted) open pull-through colectomy. ${ }^{36-38}$ This algorithm provides a simple and useful tool for HD investigation and reduces unnecessary CE-attributable delay in diagnosis and unnecessary exposure to radiation. Therefore, we suggest its clinical implementation.

However, our study has some limitations. The first is that despite the large original patient cohort $(n=107)$, only 32 of these patients had confirmed HD and only 2 patients had longer segment HD $(n=2)$. Therefore, the ability to detect differences between radiological and histological level of aganglionosis, particularly outside the frequent location of the rectosigmoid, is limited. Furthermore, our data analysis is based on a preselected patient group who had rectal biopsies and it cannot be determined how many negative CE were never referred for biopsies. Thus, this portion of CE cannot be included in the present evaluation, representing a possible bias. A false diagnosis or inconclusive CE may be attributable to technical factors, such as the applied variable contrast medium volume, instillation pressure, and level of insertion of the rectal catheter, despite a standardized approach. Furthermore, there is the possibility of misdiagnosis due to a complete aganglionosis with no evident caliber change in the CE. Moreover, due to the retrospective nature of the study, various biases can affect the data quality, such as undetectable confounding factors. Further studies may address these limitations. However, this study points to the need for a strict approach in HD diagnostics and indication for $\mathrm{CE}$ to avoid unnecessary diagnostic interventions and radiation exposure.

\section{Conclusion}

Different approaches can be used for HD diagnostic in chronic constipated children, and we aimed to evaluate the diagnostic accuracy of a CE as the first diagnostic for HD in our cohort of chronic constipated children. Based on the data of our study, we suggest that children with a high suspicion for HD, defined by medical history and clinical examination, should be first diagnosed for HD by rectal biopsies and that a CE should only be performed for subsequent diagnostic and surgical planning only in cases of previous histological confirmation of HD. This reasonable indication for $\mathrm{CE}$ reduces unnecessary CE-attributable delay in diagnosis and unnecessary exposure to radiation, without any impairment of diagnostic accuracy for HD ( - Fig. 4).

\section{Conflict of Interest}

The authors declare no conflict of interest.
Financial Support

No financial support was provided.

\section{References}

1 Haricharan RN, Seo JM, Kelly DR, et al. Older age at diagnosis of Hirschsprung disease decreases risk of postoperative enterocolitis, but resection of additional ganglionated bowel does not. J Pediatr Surg 2008;43(6):1115-1123

2 White FV, Langer JC. Circumferential distribution of ganglion cells in the transition zone of children with Hirschsprung disease. Pediatr Dev Pathol 2000;3(3):216-222

3 Lewis NA, Levitt MA, Zallen GS, et al. Diagnosing Hirschsprung's disease: increasing the odds of a positive rectal biopsy result. J Pediatr Surg 2003;38(3):412-416, discussion 412-416

4 Martucciello G, Pini Prato A, Puri P, et al. Controversies concerning diagnostic guidelines for anomalies of the enteric nervous system: a report from the fourth International Symposium on Hirschsprung's disease and related neurocristopathies. J Pediatr Surg 2005;40(10):1527-1531

5 Moore SW, Johnson G. Acetylcholinesterase in Hirschsprung's disease. Pediatr Surg Int 2005;21(4):255-263

6 Das K, Kini U, Babu MK, Mohanty S, D'Cruz AJ. The distal level of normally innervated bowel in long segment colonic Hirschsprung's disease. Pediatr Surg Int 2010;26(6):593-599

7 de Lorijn F, Kremer LC, Reitsma JB, Benninga MA. Diagnostic tests in Hirschsprung disease: a systematic review. J Pediatr Gastroenterol Nutr 2006;42(5):496-505

8 Diamond IR, Casadiego G, Traubici J, Langer JC, Wales PW. The contrast enema for Hirschsprung disease: predictors of a falsepositive result. J Pediatr Surg 2007;42(5):792-795

9 Noviello C, Cobellis G, Romano M, Amici G, Martino A. Diagnosis of Hirschsprung's Disease: an age-related approach in children below or above one year. Colorectal Dis 2010;12(10):1044-1048

10 Garcia R, Arcement C, Hormaza L, et al. Use of the recto-sigmoid index to diagnose Hirschsprung's disease. Clin Pediatr (Phila) 2007;46(1):59-63

11 Pochaczevsky R, Leonidas JC. The "recto-sigmoid index". A measurement for the early diagnosis of Hirschsprung's disease. Am J Roentgenol Radium Ther Nucl Med 1975;123(4):770-777

12 Seigel DG, Podgor MJ, Remaley NA. Acceptable values of kappa for comparison of two groups. Am J Epidemiol 1992;135(5):571-578

13 De la Torre-Mondragón L, Ortega-Salgado JA. Transanal endorectal pull-through for Hirschsprung's disease. J Pediatr Surg 1998; 33(8):1283-1286

14 De La Torre L, Langer JC. Transanal endorectal pull-through for Hirschsprung disease: technique, controversies, pearls, pitfalls, and an organized approach to the management of postoperative obstructive symptoms. Semin Pediatr Surg 2010;19(2):96-106

15 Maerzheuser S, Bassir C, Rothe K. Hirschsprung disease in the older child: diagnostic strategies. Clin Pediatr (Phila) 2012;51(11): 1087-1090

16 Reid JR, Buonomo C, Moreira C, Kozakevich H, Nurko SJ. The barium enema in constipation: comparison with rectal manometry and biopsy to exclude Hirschsprung's disease after the neonatal period. Pediatr Radiol 2000;30(10):681-684

17 Stranzinger E, DiPietro MA, Teitelbaum DH, Strouse PJ. Imaging of total colonic Hirschsprung disease. Pediatr Radiol 2008;38(11): $1162-1170$

18 AWMF online. Available at: http://www.awmf.org/leitlinien/ detail/11/006-001.html. Accessed March 16, 2015

19 Esayias W, Hawaz Y, Dejene B, et al. Barium enema with reference to rectal biopsy for the diagnosis and exclusion of Hirschsprung disease. East Cent Afr J Surg 2013;18:141-145

20 Smith GH, Cass D. Infantile Hirschsprung's disease, is a barium enema useful? Pediatr Surg Int 1991;6:318-321 
21 Swenson O, Sherman JO, Fisher JH. Diagnosis of congenital megacolon: an analysis of 501 patients. J Pediatr Surg 1973;8(5):587-594

22 Taxman TL, Yulish BS, Rothstein FC. How useful is the barium enema in the diagnosis of infantile Hirschsprung's disease? Am J Dis Child 1986;140(9):881-884

23 Pini-Prato A, Avanzini S, Gentilino V, et al. Rectal suction biopsy in the workup of childhood chronic constipation: indications and diagnostic value. Pediatr Surg Int 2007;23(2):117-122

24 Alizai NK, Batcup G, Dixon MF, Stringer MD. Rectal biopsy for Hirschsprung's disease: what is the optimum method? Pediatr Surg Int 1998;13(2-3):121-124

25 Ghosh A, Griffiths DM. Rectal biopsy in the investigation of constipation. Arch Dis Child 1998;79(3):266-268

26 Rahman N, Chouhan J, Gould S, et al. Rectal biopsy for Hirschsprung's disease-are we performing too many? Eur J Pediatr Surg 2010;20(2):95-97

27 Langer JC. Laparoscopic and transanal pull-through for Hirschsprung disease. Semin Pediatr Surg 2012;21(4):283-290

28 Knowles $\mathrm{CH}$, De Giorgio R, Kapur RP, et al. Gastrointestinal neuromuscular pathology: guidelines for histological techniques and reporting on behalf of the Gastro 2009 International Working Group. Acta Neuropathol 2009;118(2):271-301

29 Aldridge RT, Campbell PE. Ganglion cell distribution in the normal rectum and anal canal. A basis for the diagnosis of Hirschsprung's disease by anorectal biopsy. J Pediatr Surg 1968;3(4):475-490

30 Tafazzoli K, Soost K, Wessel L, Wedel T. Topographic peculiarities of the submucous plexus in the human anorectum-consequences for histopathologic evaluation of rectal biopsies. Eur J Pediatr Surg 2005;15(3):159-163

31 Athow AC, Filipe MI, Drake DP. Problems and advantages of acetylcholinesterase histochemistry of rectal suction biopsies in the diagnosis of Hirschsprung's disease. J Pediatr Surg 1990;25(5):520-526

32 Dobbins WO III, Bill AH Jr. Diagnosis of Hirschsprung's disease excluded by rectal suction biopsy. N Engl J Med 1965; 272:990-993

33 Jamieson DH, Dundas SE, Belushi SA, Cooper M, Blair GK. Does the transition zone reliably delineate aganglionic bowel in Hirschsprung's disease? Pediatr Radiol 2004;34(10):811-815

34 Proctor ML, Traubici J, Langer JC, et al. Correlation between radiographic transition zone and level of aganglionosis in Hirschsprung's disease: Implications for surgical approach. J Pediatr Surg 2003;38(5):775-778

35 De Campo JF, Mayne V, Boldt DW, De Campo M. Radiological findings in total aganglionosis coli. Pediatr Radiol 1984;14(4):205-209

36 Marquez TT, Acton RD, Hess DJ, Duval S, Saltzman DA. Comprehensive review of procedures for total colonic aganglionosis. J Pediatr Surg 2009;44(1):257-265, discussion 265

37 Miyano G, Ochi T, Lane GJ, Okazaki T, Yamataka A. Factors affected by surgical technique when treating total colonic aganglionosis: laparoscopy-assisted versus open surgery. Pediatr Surg Int 2013; 29(4):349-352

38 Muensterer OJ, Chong A, Hansen EN, Georgeson KE. Single-incision laparoscopic endorectal pull-through (SILEP) for Hirschsprung disease. J Gastrointest Surg 2010;14(12):1950-1954 